\title{
METODOLOGIAS DE SÍNTESE DE 2-ARILCICLOEXANONAS
}

\section{Roberto P. Santos}

Universidade Estácio de Sá, Rua do Bispo, 83, 20261-060 Rio de Janeiro - RJ

Mauro B. de Amorim

Núcleo de Pesquisas de Produtos Naturais, Centro de Ciências da Saúde, Universidade Federal do Rio de Janeiro, 21941-590

Rio de Janeiro - RJ

Rosangela S. C. Lopes e Claudio C. Lopes*

Departamento de Química Analítica, Instituto de Química, Centro de Tecnologia, Universidade Federal do Rio de Janeiro, 21949- 900 Rio de Janeiro - RJ

Recebido em 20/12/01; aceito em 18/10/02

\begin{abstract}
METHODOLOGIES OF SYNTHESIS OF 2-ARYLCYCLOHEXANONES. Several methodologies concerning the preparation of 2-aryl and 2-heteroarylcyclohexanones are presented. The use of these intermediates in the synthesis of chemically and biologically interesting organic compounds is also discussed.
\end{abstract}

Keywords: 2-arylcyclohexanones; 2-heteroarylcyclohexanones; cyclohexanone; $\alpha$-arylketones.

\section{INTRODUÇÃO}

2-arilcicloexanonas e 2-heteroarilcicloexanonas formam uma classe de compostos utilizados com relativa frequiência na obtenção de moléculas de interesse químico e biológico. Apesar disto, são poucas as metodologias gerais para obtenção destes intermediários restringindo-se, em sua maioria, à preparação de apenas alguns membros desta classe.

Devido ao interesse em se ter acesso a informações resumidas que possam auxiliar tanto na obtenção destes intermediários como na aplicação dos mesmos na síntese de outras substâncias, apresentamos nesta revisão os métodos de preparação mais comuns, seguindo-se alguns exemplos da aplicação destes intermediários na elaboração de substâncias estruturalmente mais sofisticadas.

\section{MÉTODOS DE SÍNTESE}

Os métodos de síntese de 2-arilcicloexanonas mais difundidos são os de autoria de Newman-Farbman e Wildman-Wildman. Outros métodos, por diferentes abordagens, também conduzem a estas substâncias, embora tenham se restringido à obtenção de apenas alguns exemplos, não se constituindo em metodologias gerais de obtenção. Apresentamos, nesta parte, uma descrição resumida dos principais métodos de preparação encontrados na literatura.

\section{Método de Newman-Farbman ${ }^{1}$}

O método de Newman-Farbman consiste na reação de um reagente de Grignard preparado a partir de um haleto aromático convenientemente substituído (1) com $\alpha$-clorocicloexanona (2), fornecendo a 2-arilcicloexanona desejada em uma única etapa ${ }^{2,3}$. Os rendimentos de 2-arilcicloexanonas obtidas por este processo oscilam em torno de $50 \%$, encontrando aplicação na síntese de vários intermediários, apesar das limitações encontradas quando a porção alicíclica apresenta substituintes. Por exemplo, a adição de brometo de fenil-magnésio (1a) à 2-cloro-4-metilcicloexanona (4) resulta em uma mistura de 4- (5) e 5-metil-2-fenilcicloexanonas (6, Esquema 1) ${ }^{4}$.

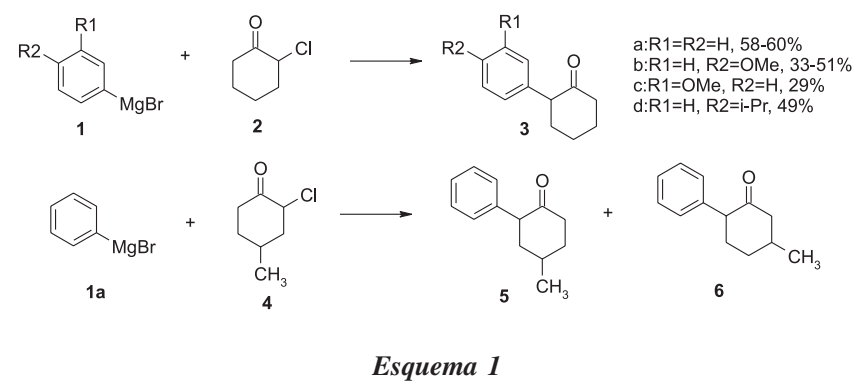

A proposta mecanística mais coerente para estes resultados experimentais aponta para o ataque inicial do organo-metálico 1a à carbonila, gerando o alcóxido $\mathbf{8}$ que, por substituição nucleofílica interna, desloca o halogênio, formando o intermediário epóxido $\mathbf{9}$. No processo de isolamento, há a abertura do anel epóxido, com formação do carbênio benzílico $\mathbf{1 0}$ que, por eliminação, fornece a 2fenilcicloexanona (3a), via o enol 11. (Esquema 2).

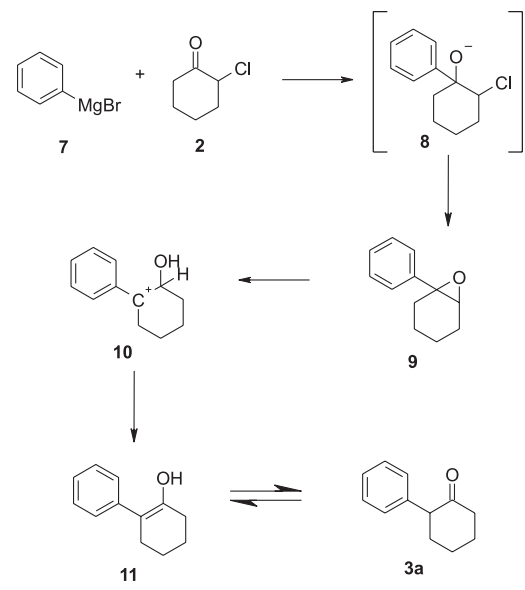

Esquema 2 


\section{Método de Wildman-Wildman ${ }^{4}$}

O método de Wildman-Wildman consiste em uma modificação de um processo concebido anteriormente por Barltrop-Nicholson ${ }^{5}$. A seqüência de reações proposta orginalmente por Barltrop-Nicholson inicia-se com a reação de Diels-Alder entre 1,3-butadieno (12) e um $\beta$-nitroestireno (13). O 4-nitro-5-arilcicloexeno (14) obtido é submetido à hidrogenação catalítica, fornecendo o 2-nitrocicloexilareno $\mathbf{1 6}$. Este, então, submetido às condições da reação de Nef (hidrólise ácida do nitronato), leva à 2-arilcicloexanona desejada. $\mathrm{O}$ método de Wildman-Wildman, que proporciona melhores rendimentos, inverte as etapas de hidrólise do nitronato e hidrogenação. Na primeira etapa, obtém-se a 6-aril-2-cicloexen-1-ona 15 que, hidrogenada, proporciona a 2-arilcicloexanona (Esquema 3 ).

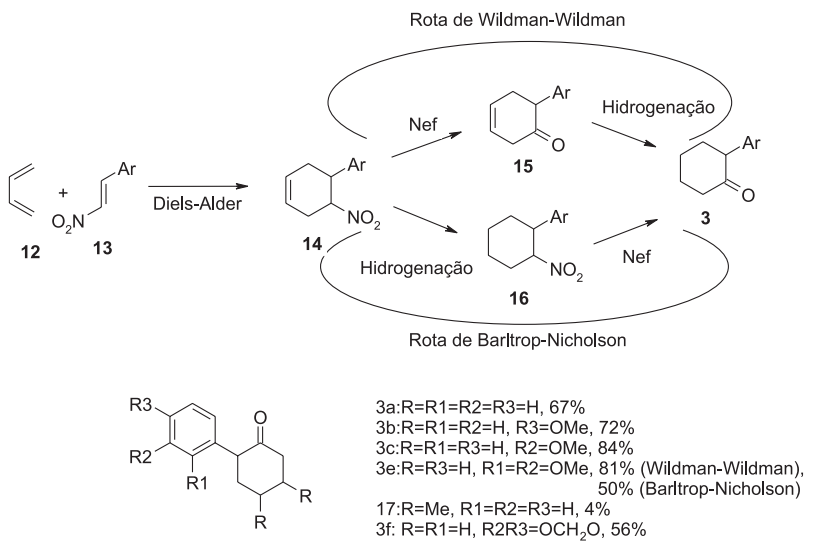

Esquema 3

Este processo permite a obtenção de várias 2-arilcicloexanonas, com bons rendimentos. Entretanto, cabe ressaltar que tal metodologia não elimina a dificuldade de se manipular de forma conveniente a substituição na porção alicíclica. A reação de Diels-Alder, etapachave na formação desta parte da molécula, pode levar à formação de isômeros, quando o dieno ou dienófilo apresentam substituintes em suas cadeias ${ }^{6}$.

\section{Método de Price-Karabinos ${ }^{7}$}

É um método de aplicação restrita, utilizado em estudos sobre a desidratação de cicloexanóis, na década de quarenta. O método consiste na hidrogenação catalítica de um orto-arilfenol, como $\mathbf{1 8}$, que leva ao 2-fenilcicloexanol (19) o qual, por oxidação, fornece a 2fenilcicloexanona (3a) (Esquema 4). O rendimento global apresentado pelos autores é de $60 \%$ para a 2 -fenilcicloexanona, e não temos conhecimento de trabalhos em que conste a utilização desta metodologia para preparação de outros intermediários. A preparação do orto-arilfenol com substituintes na porção relacionada ao grupo aril da molécula de 2-arilcicloexanona constitui o principal obstáculo à utilização desta sequiência de reações.

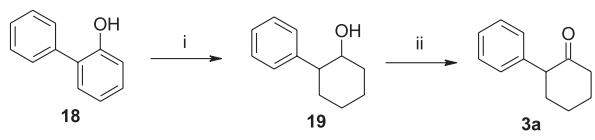

Esquema 4. $\mathrm{i}: \mathrm{H}_{2} / \mathrm{Ni}, 150^{\circ} \mathrm{C}, 75 \%$; $\mathrm{ii}: \mathrm{CrO}_{3}, 80 \%$

\section{Método de Horning}

O processo de Horning, desenvolvido na década de 40, foi utilizado, ainda que pouco freqüentemente, na preparação de unidades octaidrofenantrênicas. Consiste na alquilação de uma $\alpha$ arilacetonitrila (20) com $\delta$-clorovaleronitrila, utilizando-se $\mathrm{NaNH}_{2}$ como base. A $\alpha$-arilpimelonitrila (21) resultante é ciclizada à $\alpha$-ciano$\alpha$-arilcicloexilimina 22, que após hidrólise, fornece a 2-arilcicloexanona correspondente (3e), com rendimentos da ordem de $11 \%$ (Esquema 5).

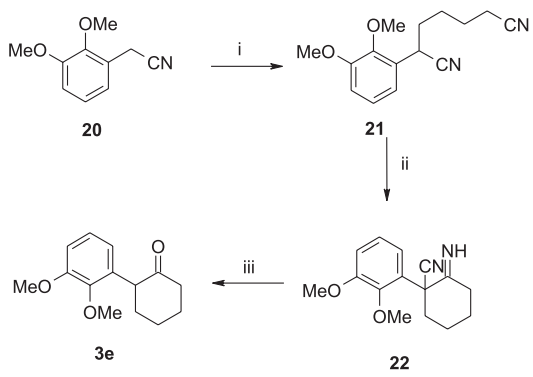

Esquema 5. i: $\mathrm{NaNH}_{2}, \mathrm{Cl}\left(\mathrm{CH}_{2}\right)_{4} \mathrm{CN}, 43 \%$; ii: $\mathrm{Na}, 45 \%$; iii: $\mathrm{HCl}, \mathrm{MeOH}$, $57 \%$

\section{Método de Bergman?}

Bergman e colaboradores relataram a preparação da 2-(2,3dimetoxifenil)cicloexanona (3e) por duas rotas alternativas. A primeira rota utiliza a adição do organo-lítio $\mathbf{2 3}$ ao óxido de cicloexeno, fornecendo o álcool $\mathbf{2 4}$ que, após oxidação, conduz a 2-arilcicloexanona 3e. A segunda rota utiliza a adição de $\mathbf{2 3}$ à cicloexanona, proporcionando o álcool benzílico 25 que, desidratado, gera a olefina 26. A hidroxilação com perácido ou tetróxido de ósmio fornece o cis-diol 27, que sofre desidratação e enolização, fornecendo a arilcicloexanona 3e (Esquema 6).

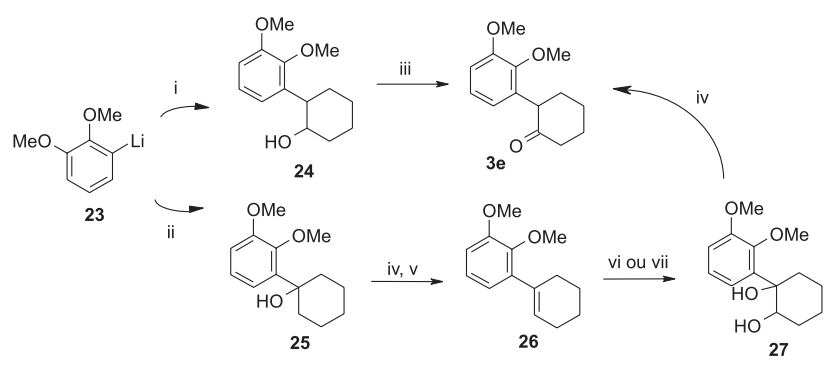

Esquema 6. $i$ : óxido de cicloexeno, $47 \%$; ii: cicloexanona, $52 \%$; iii: $\mathrm{CrO}_{3}$, $\mathrm{AcOH}, 90 \%$; iv: HOOC-COOH, 70\%; v: $\triangle$, tolueno, $62 \%$; vi: $\mathrm{HCOOH}$, $\mathrm{H}_{2} \mathrm{O}_{2}, 71 \%$; vii: $\mathrm{OsO}_{4}, 30 \%$

O rendimento global, em ambos os casos, é de no máximo $22 \%$. Entretanto, a adição ao óxido de cicloexeno seguida de oxidação do álcool intermediário com PDC é um método relatado para a formação de 2-arilcicloexanonas com substituintes aril policíclicos ${ }^{10}$. A oxidação do álcool com ácido peroxiacético e o éster de cromo 75 em quantidade catalítica forneceu a 2 -fenilcicloexanona ${ }^{11}$, com excelente rendimento.

Como alternativa, a conversão da olefina 26 à 2-arilcicloexanona poderia ser realizada por hidroboração seguida de oxidação ${ }^{12}$. 


\section{Método de Caubere-Noguchi ${ }^{13,14}$}

Caubere e colaboradores utilizaram a condensação de benzinos com enolatos de cicloalcanonas, apresentando uma elegante alternativa para a preparação de 2-arilcicloexanonas. Tal método é objeto de uma patente, de autoria de Noguchi e colaboradores, estendendo o trabalho à preparação de outras 2-arilcicloexanonas ${ }^{15}$.

$\mathrm{O}$ método de Caubere consiste no tratamento de um brometo de arila com amideto de sódio e/ou ter-butóxido de potássio na presença de um enolato de cicloexanona, em THF ou DME. O método de Noguchi e colaboradores diferencia-se pelo emprego, além do enolato de cicloexanona, de suas enaminas obtidas da reação com morfolina, com piperidina, com pirrolidina ou com metilanilina. Neste método, pode-se empregar, além do brometo de arila, o correspondente cloreto ou o $o$-benziléter. Os rendimentos obtidos são, em média, de $60 \%$. Entretanto, o método não foi estendido a 2-arilcicloexanonas parasubstituídas (Tabela 1).

Tabela 1. 2-Arilcicloexanonas pelo método de Caubere-Noguchi ${ }^{15}$

Cetona Rendimento em THF Rendimento em DME<smiles>O=C1CCCCC1c1ccccc1</smiles>

3a<smiles>COc1cccc(C2CCCCC2=O)c1</smiles>

$60-70 \%$

$0 \%$<smiles>CC1(c2ccccc2)CCCCC1=O</smiles>
$58 \%$ $90 \%$

31<smiles>CC1CCCC(C)(c2ccccc2)C1=O</smiles>

$3 \mathrm{~m}$

\section{Método de Cantacuzene ${ }^{16}$}

Reagindo uma enamina da 2-clorocicloexanona (halogênio em posição alílica) com um organometálico $(\mathrm{Mg}, \mathrm{Li}$ ou $\mathrm{Cu})$ conforme apresentado no Esquema 7, Cantacuzene obteve várias 2arilcicloexanonas, com rendimentos numa faixa de 18 a $85 \%$, variando de acordo com o organometálico, o solvente e a enamina em-

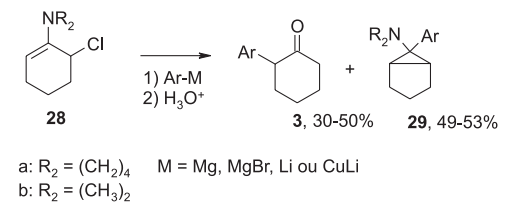

pregados. A Tabela 2 compara os rendimentos para a reação da enamina 28a com diversos organometálicos em THF e éter ${ }^{16}$.

É interessante observar as diferenças entre os rendimentos obtidos com a utilização das enaminas originárias da dimetilamina (28b) e da pirrolidona (28a). Por exemplo, utilizando brometo de fenilmagnésio e $\mathbf{2 8 b}$ em éter etílico, a 2-fenilcicloexanona não foi obtida, enquanto que empregando 28 a o rendimento foi de $50 \%$.

A diminuição do rendimento das cetonas por este método é acompanhada do aumento da produção do produto bicíclico 29. Observase que grupos retiradores de elétrons presentes em posição para- no anel aromático favorecem a formação da 2-arilcicloexanona, enquanto grupos doadores de elétrons nesta posição favorecem a formação do produto bicíclico 29 (compare as entradas 6 a 11 da Tabela 2).

Tabela 2. 2-Arilcicloexanonas pelo método de Cantacuzene ${ }^{16}$

\begin{tabular}{ccccc}
\hline Entrada & $\mathrm{Ar}-\mathrm{M}$ & Solvente & $\% \mathbf{3}$ & \% 29 \\
\hline 1 & $\mathrm{PhMgBr}$ & $\mathrm{Et}_{2} \mathrm{O}$ & 50 & 49 \\
2 & $\mathrm{Ph}{ }_{2} \mathrm{Mg}$ & $\mathrm{Et}_{2} \mathrm{O}$ & 50 & 49 \\
3 & $\mathrm{PhMgBr}$ & $\mathrm{THF}$ & 30 & 47 \\
4 & $\mathrm{PhLi}$ & $\mathrm{Et}_{2} \mathrm{O}$ & 45 & 53 \\
5 & $\mathrm{Ph}_{2} \mathrm{CuLi}$ & $\mathrm{Et}_{2} \mathrm{O}$ & 38 & 0 \\
6 & $\mathrm{p}-\mathrm{CH}_{3} \mathrm{OPhMgBr}$ & $\mathrm{Et}_{2} \mathrm{O}$ & 37 & 62 \\
7 & $\left(\mathrm{p}-\mathrm{CH}_{3} \mathrm{OPh}\right)_{2} \mathrm{Mg}$ & $\mathrm{Et}_{2} \mathrm{O}$ & 18 & 82 \\
8 & $\mathrm{p}-\mathrm{CH}_{3} \mathrm{OPhMgBr}$ & $\mathrm{THF}_{3}$ & 35 & 22 \\
9 & $\mathrm{p}-\mathrm{CF}_{3} \mathrm{PhMgBr}$ & $\mathrm{Et}_{2} \mathrm{O}$ & 80 & 19 \\
10 & $\left(\mathrm{p}-\mathrm{CF}_{3} \mathrm{Ph}\right)_{2} \mathrm{Mg}$ & $\mathrm{Et}_{2} \mathrm{O}$ & 85 & 12 \\
11 & $\mathrm{p}-\mathrm{CF}_{3} \mathrm{PhMgBr}$ & $\mathrm{THF}$ & 60 & 12 \\
\hline
\end{tabular}

\section{Método de Ireland ${ }^{17}$}

O método de Ireland consiste na reação entre um cátion organometálico do tipo penta-hapto-cicloexadienilferro tricarbonil (30) com uma enamina (31). Embora aplicado apenas na preparação de $\mathbf{3 b}$, este método poderia ser utilizado na preparação de 2arilcicloexanonas, apesar do processo apresentar um rendimento global de apenas 29\%, a partir do cátion 30 (Esquema 8).

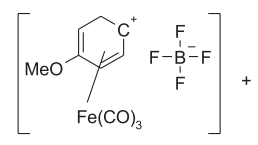

30

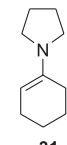

31

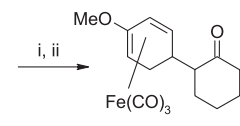

32

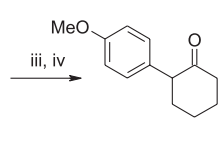

3b
Esquema 8. $\mathrm{i}: \mathrm{CH}_{2} \mathrm{Cl}_{2}$; ii: $\mathrm{H}_{3} \mathrm{O}^{+}, 69 \%$; iii: $\mathrm{Ce}^{\mathrm{IV}} ; \mathrm{iv}: \mathrm{DDQ}, 42 \%$

\section{Método de Sacks-Fuchs ${ }^{18}$}

A reação de adição do tipo Michael de difenilcuprato de lítio ou fenilcobre a $p$-toluenosulfonilazocicloex-1-eno (33), produz a $\alpha$ feniltosilhidrazona correspondente (34). $\mathrm{O}$ tratamento deste produto com $\mathrm{BF}_{3} \cdot \mathrm{Et}_{2} \mathrm{O}$ em acetona/água permite a obtenção de 2fenilcicloexanona com rendimento de $75 \%$ (Esquema 9).

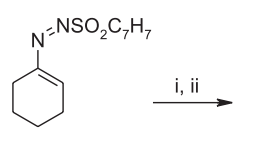

33

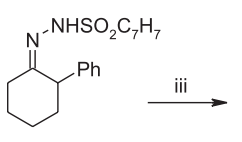

34

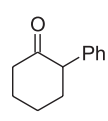

$3 a$
Esquema 9. $\mathrm{i}$ : $\mathrm{Ph}{ }_{2} \mathrm{CuLi}$ ou PhCu; ii: $\mathrm{H}_{3} \mathrm{O}^{+}, 70-75 \%$; iii: $\mathrm{BF}_{3}$. $\mathrm{Et}_{2} \mathrm{O},\left(\mathrm{CH}_{3}\right)_{2} \mathrm{CO}$, $\mathrm{H}_{2} \mathrm{O}, 94 \%$ 


\section{Método de Umezawa ${ }^{19}$}

A reação entre cicloexanona (36) e um reagente de Grignard (35) seguida de desidratação, hidroboração e oxidação fornece arilcetonas em cerca de $46 \%$ (Esquema 10). Este procedimento é uma variação do procedimento de Bergman (vide Esquema 6).
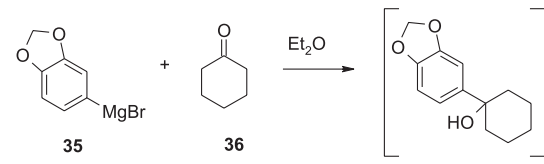

37

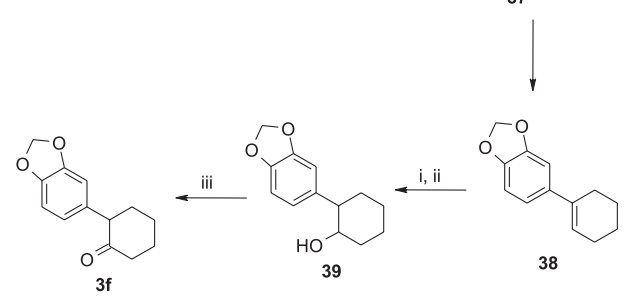

Esquema 10. $\mathrm{i}: \mathrm{NaBH}_{4} / \mathrm{BF}_{3}$; ii: $\mathrm{H}_{2} \mathrm{O}_{2}, 76 \%$; iii: $\mathrm{CrO}_{3} / \mathrm{Py}, 85 \%$

\section{Método de Barton ${ }^{20}$}

O método de Barton consiste na reação de triarilbismutocarbonatos (40) com 2-carboetoxicicloexanona (41). A reação envolve a arilação da posição $\alpha$-carbonila, onde o primeiro passo caracteriza-se pela formação de um intermediário covalente bismutooxigênio (42), seguida da formação da ligação carbono-carbono em posição ipso, concomitantemente à eliminação redutiva da porção organo-bismuto (Esquema 11). Os rendimentos das 2-aril-2carboetoxicicloexanonas resultantes (43) situam-se na faixa de 80 a $90 \%$. Apesar dos bons rendimentos, não foram sintetizadas substâncias com substituintes em posição orto- ou meta- do anel aromático. Opcionalmente, a arilação do $\beta$-cetoéster $\mathbf{4 1}$ pode ser realizada com a utilização de triacetatos de aril-chumbo ${ }^{21}$.

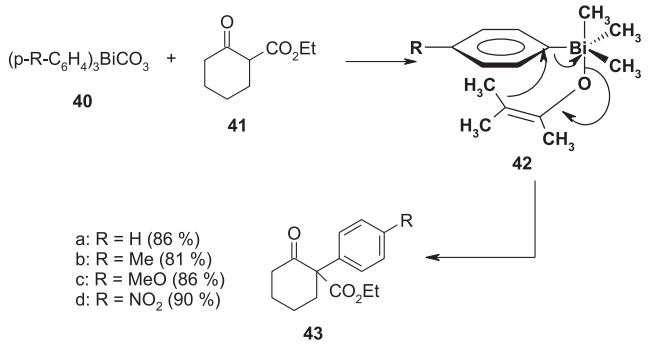

Esquema 11

\section{Método de Rathke ${ }^{22}$}

O protocolo de Rathke consiste na bromação da enamina obtida da reação de cicloexanona com morfolina e posterior reação do brometo obtido com um organocuprato, seguida de hidrólise. Os rendimentos dos regioisômeros obtidos podem variar de acordo com o organo-metálico utilizado. Por exemplo, a reação de uma mistura de 44 e 45 com difenilcupratos de lítio fornece as cetonas 31 e 46 em rendimentos de 17 e $68 \%$ e traços do produto lateral 47 , respectivamente. Com a utilização de fenilcobre, entretanto, os rendimentos são de 54 e 18\%. O processo é ilustrado no Esquema 12. Apesar dos rendimentos obtidos serem regulares, o método não foi estendido a outras 2-arilcicloexanonas.
Uma alternativa à utilização dos difenilcupratos é a substituição dos mesmos por triacetatos de aril-chumbo, embora esta variação encontre limitações devido à possibilidade de acetoxilação ${ }^{23}$.

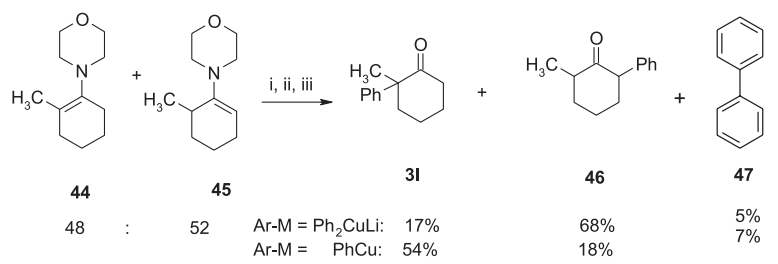

Esquema 12. $i$ : NBS; ii: $\mathrm{Ar}-\mathrm{M}$; iii: $\mathrm{H}_{3} \mathrm{O}^{+}$

\section{Método de $\mathrm{Oh}^{24}$}

A reação entre o 1-nitro-1-cicloexeno (49) e compostos aromáticos na presença de cloreto de titânio (IV) (Esquema 13) constitui o método de Oh. A reação fornece diretamente as 2-arilcicloexanonas correspondentes, uma vez que os nitronatos intermediários (50) são hidrolisados no processo de isolamento. Este método apresenta rendimentos que oscilam entre 72 e $94 \%$, sendo utilizado também para a obtenção de heteroaril (furil) cicloexanonas. A limitação do método, entretanto, reside em que apenas podem ser sintetizadas 2arilcicloexanonas com substituintes em posição para- na porção aromática. Além disso, grupos sensíveis à ação de ácidos de Lewis, como o metilenodioxi, podem sofrer ruptura no decorrer da reação ${ }^{25}$.

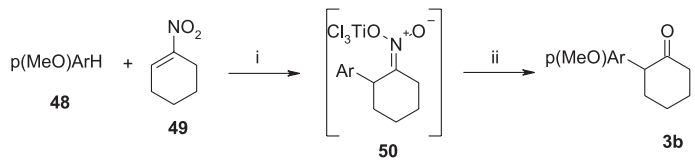

Esquema 13. $\mathrm{i}: \mathrm{TiCl}_{4} ; \mathrm{ii:} \mathrm{H}_{3} \mathrm{O}^{+}, 90 \%$

\section{Método de Duval ${ }^{26,27}$}

Um dos poucos processos utilizados na síntese de 2heteroarilcicloexanonas é a reação de carbometoxicicloexanonas (51) com 2,5-diidro-2,5-dimetoxifurana $(\mathbf{5 2})$, na presença de cloreto de zinco e de ácido acético, seguida da descarbometoxilação do produto obtido utilizando-se cloreto de lítio e $N$-metil-2-pirrolidona (Esquema 14).

O rendimento global para a preparação da 2-(2-furil)cicloexanona (3h) é regular (aproximadamente 42\%). Embora o método tenha sido utilizado na preparação de outras cetonas, não foi aplicado à preparação de outras 2-heteroarilcicloexanonas.

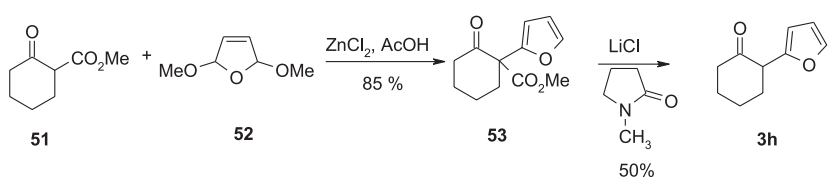

Esquema 14

\section{Método de Shudo ${ }^{28,29}$}

O protocolo de Shudo consiste na reação entre o cátion $N, N$ hidroximínio (54), derivado do 1-nitro-1-cicloexeno, com substratos aromáticos. A 2-fenilcicloexanona (3a) foi obtida por este método com um rendimento de $72 \%$ (Esquema 15). Entretanto, o processo não foi estendido a outras 2-arilcicloexanonas. 


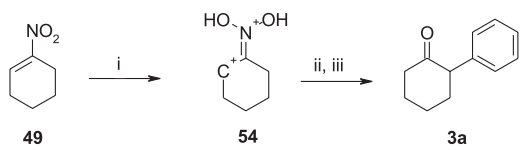

Esquema 15. $\mathrm{i}$ : TFSA; ii: $\mathrm{C}_{6} \mathrm{H}_{6},-40{ }^{\circ} \mathrm{C}$; iii: $\mathrm{MeOH}, \mathrm{H}_{2} \mathrm{O}, 72 \%$

\section{Método de $\operatorname{Koser}^{30}$}

A reação entre um enol éter de silício (55a) e fluoreto de diariliodônio (DPIF ou NPPIF ${ }^{31}$ ) foi utilizada na síntese de 2arilcicloexanonas, como $\mathbf{3} \mathbf{a}$ e $\mathbf{3 k}$, com excelentes rendimentos. Alternativamente, a reação entre triflato de difeniliodônio (DPIT) e o enolato de cobre $\mathbf{5 5}$ b pode ser utilizada na obtenção de 2fenilcicloexanona, com rendimentos de até $50 \%$ (Esquema 16) ${ }^{32}$. Apesar de ser um procedimento relativamente simples, a preparação do sal de iodônio com os grupos arila de interesse é uma etapa crucial da metodologia.

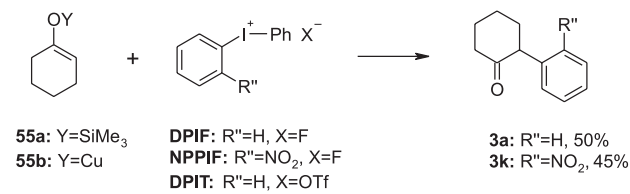

\section{Esquema 16}

\section{Método de Buchwald ${ }^{33}$}

$\mathrm{O}$ acoplamento entre um enolato de cicloexanona e um haleto arílico, na presença de complexos de paládio como catalisadores, foi utilizado na preparação de várias cetonas, como ilustrado no Esquema 17. O enolato pode ser preparado in situ, utilizando-se bases fracas, como o fosfato de potássio. Os rendimentos obtidos para as 2-arilcicloexanonas preparadas por este método situam-se entre 70 e $90 \%$ e são dependentes do tipo de haleto aromático, dos ligantes utilizados na formação do complexo e dos padrões de substituição no anel da cicloexanona e da porção aromática. Assim, a reação entre cicloexanona (36) e 56a utilizando 61 como ligante fornece a cetona 57 com rendimento de $74 \%$, enquanto a reação com $\mathbf{5 6 b}$ na presença de $\mathbf{6 2}$ leva ao produto num rendimento de $70 \%$. Entretanto, a reação da 1-tetralona $\mathbf{( 5 8 )}$ e $\mathbf{5 9}$ conduz a rendimentos diferenciados (93 e 76\%), utilizando 62 ou $\mathbf{6 3}$ como ligantes.

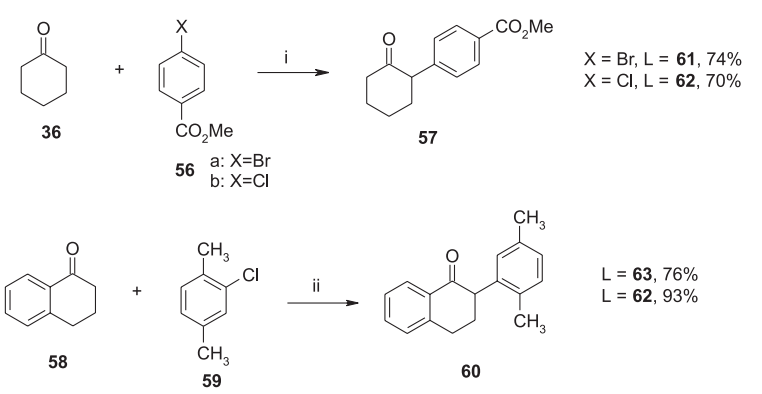

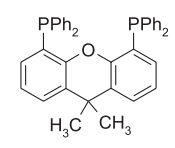

61

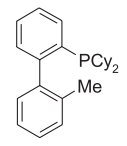

62

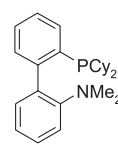

63

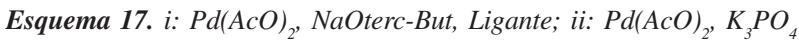

\section{Método de Nishiguchi ${ }^{34}$}

A oxidação anódica do enoléster 64 em metanol fornece o intermediário 2-metoxicicloexanona (65). A adição de um reagente de Grignard e posterior tratamento com ácido sulfúrico em diclorometano conduz às 2-arilcicloexanonas correspondentes, via 1-aril2-metoxi-1-cicloexanol.

A etapa de formação do intermediário 2-metoxicicloexanona apresenta rendimentos que dependem não apenas do padrão de substituição do anel, mas também do eletrólito utilizado. Neste aspecto, os melhores rendimentos são obtidos com o tosilato de tetraetilamônio, seguindo-se o tratamento da mistura reacional com ácido clorídrico (Esquema 18).

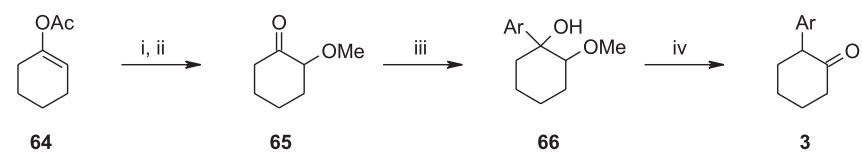

Esquema 18. $i$ : -e; $\mathrm{MeOH}, \mathrm{Et}_{4} \mathrm{NOTs}$; ii: $\mathrm{HCl}, 86 \%$; iii: $\mathrm{ArMgX}, \mathrm{THF}, 77 \%$; iv: $\mathrm{H}_{2} \mathrm{SO}_{4}, 98 \%$

\section{Método de Santos-Lopes ${ }^{35}$}

Desenvolvido recentemente, é uma metodologia geral para acesso tanto à 2-aril quanto à 2-heteroarilcicloexanonas. Consiste na adição de aril ou heteroaril-lítios ao 1-nitro-1-cicloexeno, isolando-se o intermediário 2-nitrocicloexilareno e submetendo-se o mesmo às condições da reação de Nef. Os rendimentos obtidos situam-se entre 15 e 95\%, com limitações para alguns aril-lítios (Esquema 19).

As limitações decorrem de variações no caráter nucleofílico do aril-lítio utilizado na primeira etapa do processo. O organo-metálico pode comportar-se como base, retirando um dos átomos de hidrogênio em posição alílica no 1-nitro-1-cicloexeno, o que leva à recuperação da substância após o isolamento. De acordo com estudos teóricos utilizando como modelos monômeros e dímeros de fenil-lítio, de 3-metoxifenil-lítio e de 1,3-benzodioxol-5-il-lítio, não apenas a nucleofilicidade intrínseca de cada organo-metálico, mas também a maior concentração da espécie monomérica, que possui comportamento eminentemente básico, seria responsável pela diminuição do rendimento da etapa de adição e teria, como conseqüência, a diminuição do rendimento global do processo ${ }^{36,37}$.

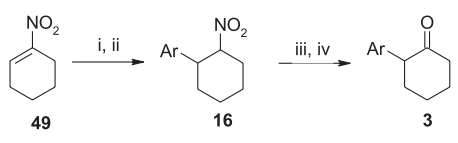

$\mathrm{a}: \mathrm{Ar}=$ fenil, $68 \%$ $\mathrm{c:Ar}=3$-metoxifenil, $15 \%$ $\mathrm{f:} \mathrm{Ar}=3,4-$ metilenodioxifenil, $95 \%$ $\mathrm{g}: \mathrm{Ar}=1$-naftil, $90 \%$ $\mathrm{h}: \mathrm{Ar}=2$-furil, $89 \%$ $\mathrm{i}: \mathrm{Ar}=2$-tienil, $88 \%$
$\mathrm{j}: \mathrm{Ar}=2$-benzofuril, $87 \%$

Esquema 19. $i$ : Ar-Li; ii: $\mathrm{AcOH}, \mathrm{O}^{\circ} \mathrm{C}$; $i i i: \mathrm{EtO}^{-} \mathrm{Na}^{+} / \mathrm{EtOH}$; iv: $\mathrm{HCl} / \mathrm{EtOH} /$ $\mathrm{H}_{2} \mathrm{O}$

\section{UTILIZAÇÃO DAS 2-ARILCICLOEXANONAS EM SÍNTESE}

Os intermediários do tipo 2-arilcicloexanonas possuem ampla aplicação na construção de moléculas naturais ou biologicamente ativas. Além disso, alguns derivados destas substâncias apresentam propriedades biológicas interessantes. Por exemplo, cicloalquilaminas (67a-c) são aplicadas no tratamento da miastenia gravis ${ }^{38}$ e os seus sais de amônio quaternários, como 68, são inibidores da acetilcolinesterase $^{39}$. Algumas cicloexilaminas substituídas (69a-f $)^{40}$ es- 
tão relacionadas às atividades estimulante do sistema nervoso central e anti-inflamatória.

A utilização de 2-arilcicloexanonas como precursores sintéticos, por sua vez, tem possibilitado a construção de esqueletos distintos. Popelak e Lettenbaurer, em 1962, utilizaram intermediários deste tipo para a construção de substâncias do tipo 9-feniloctaidroindol ${ }^{41}$. O Esquema 20(d) resume a síntese do 9-(3,4-dimetoxifenil) octaidroindol (70a), substância com ação tranquilizante. A rota sintética, utilizada por Popelak e Lettenbaurer, é uma extensão da metodologia proposta por Wildman e foi usada na comprovação das estruturas dos alcalóides crinina, powellina, bufanidrina e bufanisina. As etapas da síntese do ( \pm )-crinano (71), utilizadas na comprovação da estrutura da crinina, são apresentadas no Esquema 20(e $)^{42,43}$.

Fazendo uso de uma abordagem sintética diferenciada, porém utilizando ainda a 2-(3,4-dimetoxifenil)cicloexanona como intermediáriochave, Shamma e Rodriguez realizaram a síntese racêmica da mesembrina (70b), um alcalóide encontrado em Mesembrianthemum tortuosum. A presença de uma carbonila na posição 6 do esqueleto octaidroindólico levou ao desenvolvimento de um número consideravelmente maior de etapas na preparação do núcle ${ }^{44}$. O material de partida mais comumente empregado na síntese de estruturas com núcleo octaidroindólico é uma 2-arilcicloexanona, realizando-se adequadamente a funcionalização do intermediário em etapas posteriores. Umezawa e colaboradores utilizaram esta abordagem na preparação de licoranos, como 72, cuja rota sintética encontra-se resumida no Esquema 20(f) ${ }^{17}$.

Algumas substâncias com estruturas mais simples, porém não menos importantes com relação aos possíveis efeitos fisiológicos, como o cicloexestrol, que apresenta ação estrogênica, também podem ser preparadas a partir de 2-arilcicloexanonas. Mueller e May sintetizaram o cicloexestrol (73) utilizando a 2-(4-metoxifenil)cicloexanona (3b) como material de partida, submetendo esta substância às condições incluídas no Esquema $20(\mathrm{~g})^{2 \mathrm{a}}$.

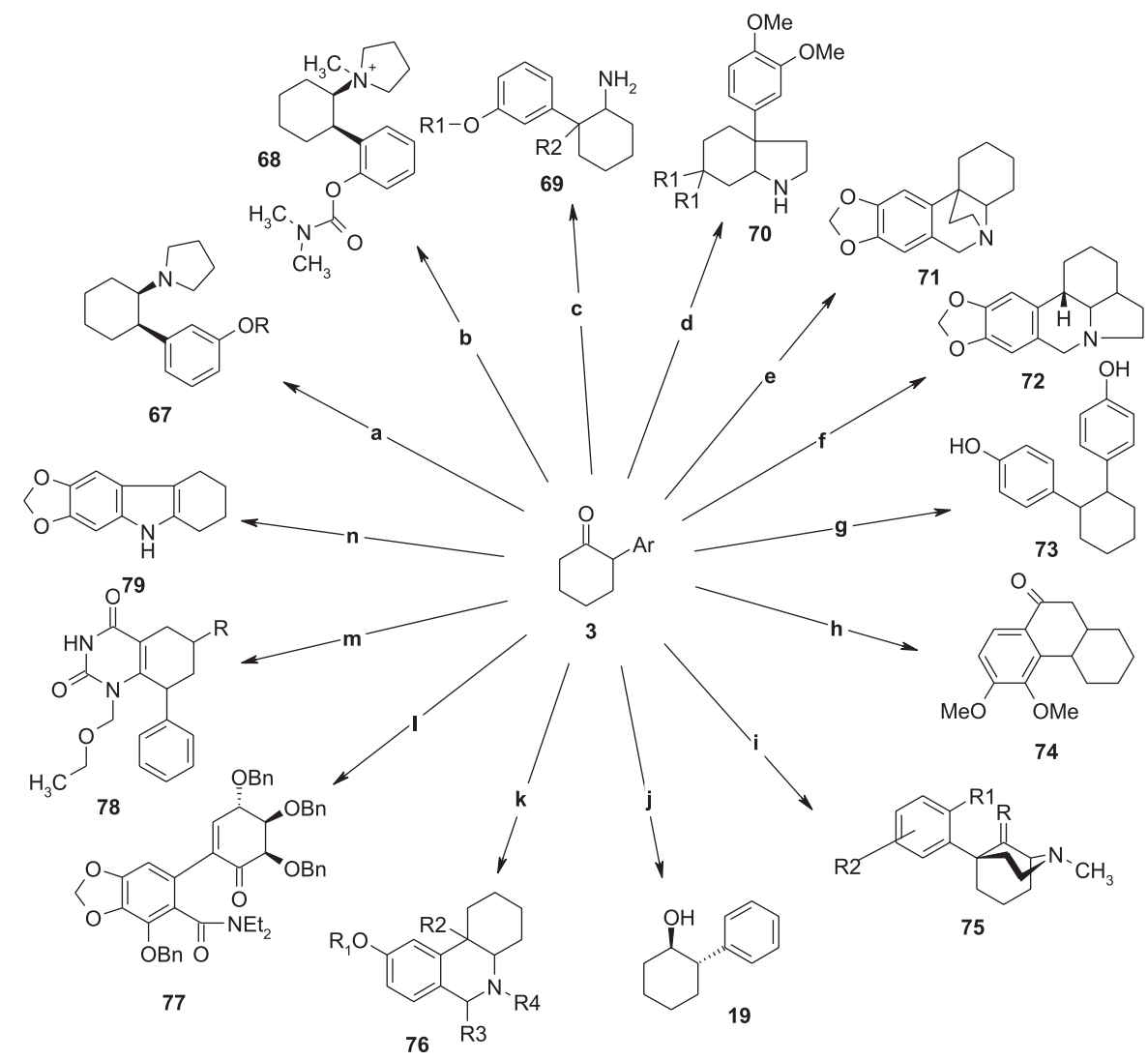

\footnotetext{
70- $\mathbf{a}: \mathrm{R} 1=\mathrm{H} ; \mathbf{b}: \mathrm{R} 1 \mathrm{R} 1=\mathrm{O}$

76- R1=H, Me, $\mathrm{CH}_{2} \mathrm{CH}_{2} \mathrm{OH}$; R2=Me, Et, $\mathrm{Pr}$, alil, ciclopr; R3=H, Me; R4=H, Me

78- a: $\mathrm{R}=\mathrm{i}-\mathrm{Pr} ; \mathbf{b}: \mathrm{R}=\mathrm{H}$

75- a: R= $\mathrm{H}_{2}, \mathrm{R} 1=\mathrm{H}, \mathrm{R} 2=3-\mathrm{OH} ; \mathbf{b}: \mathrm{R}=\mathrm{O}, \mathrm{R} 1=\mathrm{OMe}, \mathrm{R} 2=3-\mathrm{OMe}$

67- $\mathbf{a}: \mathrm{R}=\mathrm{H} ; \mathbf{b}: \mathrm{R}=\mathrm{COPr} ; \mathbf{c}: \mathrm{R}=\mathrm{CONMe}_{2}$

69- a: R1=R2=Me; b: R1=H, R2=Me; $\mathbf{~ : ~ R 1 = M e , ~ R 2 = E t ; ~}$

d: $\mathrm{R} 1=\mathrm{Me}, \mathrm{R} 2=$ ciclopr $-\mathrm{CH}_{2}$; e: $\mathrm{R} 1=\mathrm{Me}, \mathrm{R} 2=$ alil; f: $\mathrm{R} 1=\mathrm{HO}\left(\mathrm{CH}_{2}\right)_{2}, \mathrm{R} 2=\mathrm{Me}$
}

Esquema 20. (a) i: pirrolidina; ii: $\mathrm{H}_{2} / \mathrm{Ni}$-Raney. 99\%, (b) i: pirrolidina; ii: $\mathrm{H}_{2} / \mathrm{Pd}$-C; iii: $\mathrm{CH}_{3} \mathrm{I}, 87 \%$, (c) i: $\mathrm{NH}_{2} \mathrm{OH} . \mathrm{HCl} / \mathrm{EtOH} / \mathrm{Na}_{2} \mathrm{CO}{ }_{3}$; ii: $\mathrm{H}_{2} / \mathrm{Ni}$-Raney,

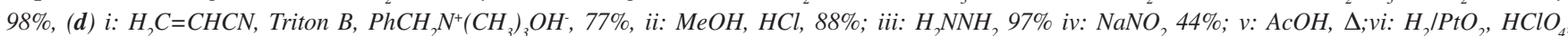
quant., (e) i: idêntico a (d); ii: $\mathrm{CH}_{2} \mathrm{O}, \mathrm{NaHCO}_{3}, \Delta, 67 \%,(f)$ i: pirrolidina, 98\% ii: $\mathrm{BrCH}_{2} \mathrm{COOCH}_{3}$, 85\%; iii: $\mathrm{KOH}, \mathrm{MeOH}, \mathrm{H}_{2} \mathrm{O}, 67 \%$; iv: $\mathrm{HCl}, \mathrm{H}$; v: $\mathrm{NH}_{2} \mathrm{OH} . \mathrm{HCl}, \mathrm{NaOH}, 92 \%$; vi: $\mathrm{Zn}, \mathrm{HOAc}$, 85\%; vii: $\mathrm{H}_{2} / \mathrm{PtO}_{2}$; viii: $\mathrm{B}_{2} \mathrm{H}_{6}$; ix: $\mathrm{CH}_{2} \mathrm{O}, 78 \%$, (g) i: $\mathrm{p}-\mathrm{MeOPhMgBr}, 93 \%$; ii: $\mathrm{HCl}$; iii: $\mathrm{H}_{2} / \mathrm{Pd}-\mathrm{C}$; iv: $\mathrm{KOH}, 88 \%$, (h) i: Reformatsky, 73\%; ii: $\mathrm{SOCl}_{2}$, quant. ; iii: $\mathrm{KOH}, 89 \%$; iv: $\mathrm{H}_{2} / \mathrm{Pd}$-C; v: $\mathrm{H}_{2} \mathrm{SO}_{4} 63 \%$, (i) i: $\mathrm{Br}_{2}, \mathrm{CHCl}_{3}$, 82\% ; ii: $\mathrm{NH}_{4} \mathrm{OH}$; iii: $\Delta$, p-xileno; iv: $\Delta, 1$-nonanol, 51\%, (j) i: MeLi, LiBr, quant. ;ii: p-TolSOCH $\mathrm{CHOHCF}_{3}$, 90\%; iii: $\alpha$-naftil-sódio, acetamida, 95\%, (k) i: $\mathrm{NaNH}_{2}$; ii: $\mathrm{CH}_{3}$ I, 77\%; iii: $\mathrm{NH}_{2} \mathrm{OH} . \mathrm{HCl}, \mathrm{AcONa}$;

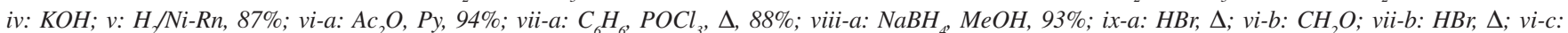
$\mathrm{CH}_{2} \mathrm{O}, 61 \%$; vii-c: $\mathrm{CH}_{2} \mathrm{O}, \mathrm{HCO}_{2} \mathrm{H}, \Delta, 72 \%$; viii-c: $\mathrm{HBr}, \Delta ; 65 \%$, (l) $\mathrm{Ac} \mathrm{O}_{2} \mathrm{O}, \mathrm{DMAP}$, piridina, $70 \%$, (m) i: ClCONCO, $30 \% ; \mathrm{ii:} 58{ }^{\circ} \mathrm{C}, 1 \mathrm{~h}$; iii: $130{ }^{\circ} \mathrm{C}, 2 \mathrm{~h}$; iv: $\mathrm{NH}_{4} \mathrm{OH}$ conc., 100\%; v: HMDS, $\left(\mathrm{NH}_{4}\right)_{2} \mathrm{SO}_{4}$; vi: TMS-triflato, $\mathrm{MeCN}, \mathrm{EtOCH}_{2} \mathrm{OEt}, 53 \%$, (n) i: $\mathrm{HNO}_{3}, \mathrm{CHCl}_{3}, 0-5{ }^{\circ} \mathrm{C}, 98 \% ; \mathrm{ii}: \mathrm{H}_{2} / \mathrm{Pd}-\mathrm{C}, \mathrm{AcOH}, 53 \%$ 
Um dos primeiros trabalhos relacionados à utilização de 2arilcicloexanonas como intermediários sintéticos foi realizado por Horning e colaboradores, na preparação da unidade metoxifenantrênica 74, a qual é encontrada em vários derivados da morfina, conforme mostra o Esquema 20(h) ${ }^{8}$. Embora a proposta de Horning e colaboradores tenha se mostrado válida para a construção da unidade metoxifenantrênica, a tentativa realizada por Barltrop e Nicholson de preparação de moléculas funcionalmente mais adequadas para a construção do esqueleto da morfina, não obteve êxito ${ }^{5}$.

O 5-(3-hidroxifenil)-2-metilmorfano (75a), substância descoberta em meados do século XX por May e Murphy, possui atividade analgésica comparável à morfina, enquanto que fenilmorfanos substituídos possuem, além da atividade analgésica, atividade antinociceptiva. Trabalhos recentes de Linders e colaboradores utilizam 2-arilcicloexanonas como intermediários-chave na síntese de compostos análogos, tais como $\mathbf{7 5 b}$, conforme apresentado no Esquema 20(i) ${ }^{45}$.

O trans-2-fenilcicloexanol (19, auxiliar de Whitesell) é um eficiente auxiliar quiral. Asensio e colaboradores, recentemente, relataram a preparação desta substância em excesso enanciomérico de $95 \%$, pela protonação enantiosseletiva do enolato de 2-fenilcicloexanona (3a) com álcoois sulfinílicos, seguida da redução diastereosseletiva da cetona formada. O Esquema 20(j) apresenta, resumidamente, estas etapas ${ }^{46}$.

As 2-arilcicloexanonas substituídas na posição 3 do grupo aril, permitem o acesso a uma variedade de núcleos fenantridínicos, como $\mathbf{7 6}^{47}$. Uma das rotas para acesso a substâncias desta classe é fornecida no Esquema 20(k) ${ }^{48}$.

Esta estratégia foi utilizada por Danishefsky e colaboradores na preparação de um possível intermediário (77) na síntese da pancratistatina, um alcalóide fenantridínico, reconhecido como potencial agente antitumoral [Esquema 20(1) $]^{49}$.

Substâncias análogas à HEPT \{1-[(2-hidroxietoxi)metil]-6(feniltio)timina , como o MKC-442, têm demonstrado alta atividade contra o HIV-1, sendo o mesmo selecionado para testes clínicos. Em recente trabalho, Pedersen e colaboradores exploraram a utilização de uma cicloexanona como intermediário sintético na preparação de um análogo (78, R=H) do $\mathrm{MKC}-442$, conforme mostrado no Esquema $20(\mathrm{~m})^{50}$.

Wenkert e Barnett verificaram que a pirólise da oxima da 2fenilcicloexanona produz, dentre outras substâncias, núcleos indólicos, como tetraidrocarbazol e carbazol, em bom rendimento ${ }^{51}$. Através de processos mais suaves, foi explorada a intermediação de 2-arilcicloexanonas no processo de formação de anéis indólicos, realizando a síntese de 6,7,8,9-tetraidro-5H[4,5-b]carbazol (79) pela seqüência de reações do Esquema 20(n) ${ }^{35}$.

\section{CONCLUSÕES}

As 2-arilcicloexanonas formam uma classe de intermediários sintéticos valiosos no acesso a esqueletos carbônicos presentes em diversas substâncias. A diferença de reatividade dos vários pontos da parte alicíclica, bem como a possibilidade de diferentes substituições no anel aromático, torna estes intermediários versatéis dos pontos de vista químico e biológico, já que modificações no esqueleto permitem facilmente a modulação da atividade. Metodologias sintéticas que viabilizem a preparação eficiente destas cetonas são importantes em Química Orgânica.

Por outro lado, possivelmente devido às limitações relacionadas à maior parte dos métodos, poucos são aqueles aplicáveis à preparação de intermediários do tipo 2-heteroarilcicloexanonas, ou mesmo 2-arilcicloexanonas com o núcleo aromático básico diferente de fenil.

\section{REFERÊNCIAS}

1. Newman, M. S.; Farbman, M. D.; J. Am. Chem. Soc. 1944, 66, 1550.

2. Mueller, G. P.; May, R.; J. Am. Chem. Soc. 1949, 71, 3313.

3. Bachman, W. E.; Fujimoto, G. I.; Wick, L. B.; J. Am. Chem. Soc. 1950, 72, 1995.

4. Wildman, W. C.; Wildman, R. B.; J. Org. Chem. 1952, 17, 581.

5. Barltrop, J. A.; Nicholson, J. S.; J. Chem. Soc. 1951, 2524.

6. March, J.; Advanced Organic Chemistry, $3^{\text {rd }}$ ed., Wiley-Interscience: New York, 1985, p. 749.

7. Price, C. C.; Karabinos, J. V.; J. Am. Chem. Soc. 1940, 62, 1159.

8. Horning, E. C.; Horning, M. G.; Platt, E. J.; J. Am. Chem. Soc. 1947, 69, 2929.

9. Bergmann, E. D.; Pappo, R.; Ginsburg, D.; J. Chem. Soc. 1950, 1369.

10. Cho, B. P.; Harvey, R. G.; Tetrahedron Lett. 1987, 28, 861.

11. Corey, E. J.; Barrette, E.-P.; Magriotis, P. A.; Tetrahedron Lett. 1985, 26, 5855.

12. Brown, H. C.; Garg, C. P.; Tetrahedron 1986, 42, 5511.

13. Caubere, P.; Gillaumet, G.; Mourad, M. S.; Tetrahedron 1972, 28, 95.

14. Caubere, P.; Mourad, M.S.; Guillaumet, G.; Tetrahedron 1973, $29,1843$.

15. Noguchi, S.; Agata, I.; Kikazawa, K.; Tsuchiki, M. ; Jpn. Kokai Tokkyo Koho 72 47, 376 1972. (CA 84:P70580u)

16. Blazejewski, J. C.; Cantacuzene, D.; Walkselman, C.; Tetrahedron 1973, 29, 4233 .

17. Ireland, R. E.; Brown Jr., G. G.; Stanford, R. H.; McKenzie, T. C.; J. Org. Chem. 1974, 39, 51 .

18. Sacks, C. E.; Fuchs, P. L.; J. Am. Chem. Soc. 1975, 97, 7372

19. Umezawa, B.; Hoshino, O.; Sawaki, S.; Sato, S.; Numao, N.; J. Org. Chem. 1977, 42, 4272.

20. Barton, D. H. R.; Bhatnagar, N. Y.; Finet, J.-P.; Motherwell, W. B.; Tetrahedron 1986, 42, 3111.

21. Abramovitch, R. A.; Barton, D. H. R.; Finet, J.-P.; Tetrahedron 1988, 44, 3039.

22. Rathke, M. W.; Vogiazoglou, D.; J. Org. Chem. 1987, 52, 3697.

23. May, G. L.; Pinhey, J. T.; Aust. J. Chem. 1982, 35, 1859.

24. Lee, K.; Oh, D. Y.; Tetrahedron Lett. 1988, 29, 2977.

25. Da Silva, A. J. M.; Tese de Mestrado, Universidade Federal do Rio de Janeiro, Brasil, 1993.

26. Duval, O.; Gomes, L. M.; Tetrahedron Lett. 1988, 29, 3243.

27. Duval, O.; Gomes, L. M.; Tetrahedron 1989, 45, 4471

28. Okabe, K.; Ohwada, T.; Ohta, T.; Shudo, K.; J. Org. Chem. 1989, 54, 733.

29. Ohwada, T.; Okabe, K.; Ohta, T.; Shudo, K.; Tetrahedron 1990, 46, 7539.

30. Chen, K.; Koser, G. F.; J. Org. Chem. 1991, 56, 5764.

31. Iwama, T.; Birman, V. B.; Kozmin, S. A.; Rawal, V. H.; Org. Lett. 1999, 1, 673.

32. Ryan, J. H.; Stang, P. J.; Tetrahedron Lett. 1997, 38, 5061.

33. Fox, J. M.; Huang, X.; Chieffi, A.; Buchwald, S. L.; J. Am. Chem. Soc. 2000, 122, 1360 e referências citadas.

34. Itoh, K.; Shino, S.; Maekawa, H.; Nishiguchi, I.; J. Eletroanal. Chem. 2001, $507,14$.

35. Santos, R. P.; Lopes, R. S. C.; Lopes, C. C.; Synthesis 2001, 845

36. Santos R. P.; Tese de Doutorado, Universidade Federal do Rio de Janeiro, Brasil, 2001.

37. Santos, R. P.; Amorim, M. B.; Lopes, C. C.; Lopes, R. S. C.; XI Simpósio Brasileiro de Química Teórica, Caxambú, Brasil, 2001.

38. Lewis, J. W.; Readhead, M. J.; Fr. Demande 2,187,296, 1974. (CA 81: 304, 3760f)

39. Lewis, J. W.; Readhead, M. J.; Ger. Offen. 2,328,896 1973. (CA 80: 348, $82362 h)$

40. F.Hoffman-La Roche \& Co., A.G., Neth. Appl. 6,609,050 1967. (CA 67: $771581942 s)$

41. Popelak, A.; Lettenbaurer, G.; US pat. 3,028,394 1962. (CA 62: 8549f)

42. Wildman, W. C.; J. Am. Chem. Soc. 1956, 78, 4180.

43. Wildman, W. C.; J. Am. Chem. Soc. 1958, 80, 2567.

44. Shamma, M.; Rodriguez, H. R.; Tetrahedron 1968, 24, 6583

45. Linders, J. T. M.; Flippen-Anderson, J. L.; George, C. F.; Rice, K. C.; Tetrahedron Lett. 1999, 40, 3905.

46. Asensio, G.; Cuenca, A.; Gaviña, P.; Medio-Simón, M.; Tetrahedron Lett. 1999, 40, 3939.

47. Hellerbach, J.; Swiss 542,848 1973. (CA 80: 338 70720k)

48. F. Hoffman-La Roche; Neth. Appl. 6,609,049 1967. (CA 67: 7740 82116n)

49. Park, T. K.; Danishefsky, S. J.; Tetrahedron Lett. 1995, 36, 195.

50. Larsen, J. S.; Christensen, L.; Ludvig, G.; Jörgensen, P. T.; Pedersen, E. B.; Nielsen, C.; J. Chem. Soc., Perkin Trans. 1 2000, 3035.

51. Wenkert, E.; Barnett, B. F.; J. Am. Chem. Soc. 1960, 82, 4671 